\title{
De la pantalla al Tweet. Ganancias y pérdidas de seguidores en los usuarios más prominentes en Twitter de América Latina y el Medio Oriente
}

\author{
Elias SAID-HuNG \\ saide@uninorte.edu.co \\ Universidad del Norte (Colombia) \\ Jorge VALENCIA CoBO \\ javalenciac@uninorte.edu.co \\ Universidad del Norte (Colombia) \\ Juan José MARTí Noguera \\ juanjomn@gmail.com \\ Universidad Antonio Nariño (Colombia)
}

Recibido: 28 de enero de 2014

Aceptado: 9 de noviembre de 2014

\begin{abstract}
Resumen
Este artículo presenta los resultados de una medición realizada a una selección usuarios de Twitter de mayor "prominencia" en países de América Latina y el Medio Oriente, así como de una muestra de los mensajes publicados por estos. Los datos mostrados aquí, fueron tomados durante el primer semestre de 2012, a partir de una investigación de corte cuantitativo, y nos ayudan a establecer los rasgos que caracterizan a los perfiles y mensajes publicados de los usuarios analizados, según las regiones tomadas en consideración; además de facilitar la identificación de factores que inciden significativamente en la ganancia y pérdida de seguidores de estos usuarios.
\end{abstract}

Palabras clave: redes sociales, mensajes, influencias, líderes, twitter.

\section{From screen to tweet. Gains and losses followers on the most prominent users of Twitter from Latin America and the Middle East}

\begin{abstract}
Our research is based a measurement made to a selection of Twitter users greater "prominence" in Latin America and the Middle East, as well as an sample of the messages posted by them. The data were taken during the first half of 2012, from a quantitative investigation of court, and was carried out to analyze for characterize the profiles and messages posted by users before mentioned, according to regions taken into consideration, in addition to providing us the identification of factors affecting significantly in the gain and loss of followers of these users.
\end{abstract}

Keywords: social media, messages, influences, leadership, twitter.

\section{Referencia normalizada}

SAID-HUNG, Elias; VALENCIA COBO, Jorge; y MARTÍ NOGUERA, Juan José (2015): "De la pantalla al Tweet. Ganancias y pérdidas de seguidores en los usuarios más prominentes en Twitter de América Latina y el Medio Oriente". Estudios sobre el Mensaje Periodístico. Vol. 21, Núm. 1 (enero-junio), págs.: 541-557. Madrid, Servicio de Publicaciones de la Universidad Complutense.

Sumario: 1. Introducción y estado de la cuestión. 2. Metdología. 3. Resultados; 3.1. Rasgos generales de los usuarios más prominentes en Twitter de América Latina y Medio Oriente; 3.2. Características de los mensajes de los usuarios más prominentes de Twitter; 3.3. Factores que inciden en la ganancia y/o pérdida de followers de los usuarios más prominentes de Twitter. 4. Discusión y conclusiones. 5. Referencias bibliográficas. 


\section{Introducción y estado de la cuestión}

El presente artículo intenta abordar diferentes dimensiones del impacto de las redes sociales, en este caso de Twitter, en lo que se refiere a la relación entre líderes de opinión y sus seguidores. Ello, sobre la base de lo expuesto por investigadores como Miller y Slater (2000), Wellman (2001), Juris (2005), Moraes (2005), Escobar (2007), Castells (2009), Jensen (2009) y Marwick y Boyd (2011), Said y Arcila (2011), Claes, Osteso y Deltell (2013), en otros; en torno a las potenciales transformaciones que se están generando en nuestras sociedades, producto de los avances de las TIC, en lo que se refiere a: 1) la forma como nos comunicamos y accedemos a información/conocimiento; 2) la creciente pérdida de marco espaciales/temporales; 3) las rutinas de acción social de cada uno de los individuos; y 4) la forma como los medios, tradicionales y nuevos, ejercen influencia, por medio de los líderes de opinión, en la apertura y seguimiento de acontecimientos sociales y, por tanto, en la construcción de opinión pública. Lo aquí expuesto se hará, desde una perspectiva de análisis centrado en el líder de opinión online dispuesto en Twitter, el cual será entendido, no solo, como aquel con más seguidores (followers); sino que cuenta con capacidad de influenciar a un mayor número de miembros de esta red social, a través de las diferentes publicaciones que comparte con el resto.

El creciente avance de las redes sociales, abre ante nosotros un contexto social y comunicativo, en el que teorías como el Two Step-Flow de Lazarsfeld, Berelson y Gaudet (1979) y las vinculadas con el estudio de los actores que influyen desde los medios de comunicación (Katz y Lazarsfeld, 1979; Rogers, 1962), más que perder vigencia, se complejizan; dando paso a planteamientos, como el propuesto por Watts y Dodds (2007), en torno a los estudios sobre los factores que inciden en la capacidad de influencia desde los nuevos medios, o Jensen (2009), en el que los escenarios digitales se convierten en ventanas por el que los líderes de opinión, tradicionales y los que surgen bajo el escenario digital/online, tienen una mayor capacidad de contacto (e influencia) con los ciudadanos (usuarios de las redes sociales). Ello al fluir la comunicación bajo la administración directa de estos, mientras los ciudadanos reciben los contenidos desde los dispositivos, por los que acceden a los diferentes escenarios digitales.

Según Túñez y Sixto (2011) las redes sociales, como resultado del volumen de participantes y la inmediatez de interacción que permiten la emisión y el control de mensajes, se establecen, no solo, en una herramienta para ganar adeptos, sino también, al momento de mantener el mensaje y la relación con los seguidores. Para que ello, "se exigen unos niveles de participación y presencia que van más allá de tener un blog o un perfil creado en una red social." (Túñez y Sixto, 2011: 4).

Bajo la perspectiva del liderazgo en las redes sociales, quienes siguen a los usuarios más prominentes/influyentes (líderes online) pueden convertirse en ciberactivistas (Olorunnisola y Martin, 2013), es decir, usuarios empoderados y agrupados alrededor determinados temas. Ello, mientras adquieren capacidad para generar un cambio social, desde la puesta en común de intereses individuales, hasta la promoción de iniciativas conjuntas para la difusión de información y el posicionamiento de tópicos en la agenda pública con una finalidad común (Ugarte, 2011). 
Tal como exponen Said-Hung y Arcila-Calderón (2011) y Deltell et al. (2013), por ejemplo, las redes sociales han conllevado la apertura de nuevos escenarios de interacción para el liderazgo, fomentando una nueva diversidad de discusiones ciudadanas, cada vez más interconectada. Tal como lo exponen estos autores, el mero hecho de que exista un seguimiento masivo a determinados individuos en las redes sociales, los convierte en potenciales líderes de opinión, ante el posible impacto que pueden traer consigo estos mensajes entre sus potenciales seguidores.

La comunicación realizada desde las redes sociales, trae consigo una mayor fluidez de mensajes multidireccionales entre usuarios-seguidores, dando mayor amplitud a los comunicados de los potenciales líderes online. Ello, como resultado de las capacidades de difusión y contacto inmediato (instantáneo) de hechos u opiniones transmitidos desde las redes sociales, a través de hashtags o el seguimiento de otros usuarios (García de Torres at al., 2011).

Las redes sociales reconfiguran la socialización y posibilitan novedosas formas de participación e interacción entre la ciudadanía; siendo uno de los resultados de este proceso la constitución de nuevas formas de relación emocional derivada de la comunicación, con base a lo expuesto por Golder y Macy (2013). La importancia creciente de redes sociales como Twitter impacta en la generación de nuevos "prosumidores" (Tofler, 1980), caracterizados por su participación directa (a través de mensajes publicados) o indirectas (desde la simple recepción y el reenvío de mensajes), en la creación de opinión pública o tendencias (trending topics).

Tal como lo expone Cha et al. (2010), el liderazgo desde las redes sociales no debería ser entendido como una cuestión accidental o espontánea, sino como un proceso en el que se concentran esfuerzos al momento de influir sobre un tópico, sin que sea tan relevante el tener un amplio número de seguidores. El concepto de líder de opinión tradicional debe repensarse desde las redes sociales y el impacto actual y creciente de estas, a nivel social. Ello pese a lo expuesto por autores como Said y Arcila (2011) quienes dejan entrever que la diferencia entre ambos tipos de líderes depende de los contextos sociales y marcos políticos y libertades democráticas existentes en los países que se analice. A pesar de ello, investigadores como Park (2013) dejan claro que lo que predomina en las redes sociales, además de la credibilidad de quien escribe, es la capacidad que se tenga al momento de demostrar experiencia y conocimiento en los temas que puedan ser abordador por estos.

Según Bakshy et al. (2011), además de entenderse el número de seguidores y tiempo de liderazgo en las redes sociales como indicadores de influencia social, existen otras variables que condicionan la capacidad de liderazgo e influencia en el tiempo, que conviene analizar con detalle. Tal como lo expone Cha et al. (2010) y Watts (2007) la prominencia que pueden tener determinados usuarios en Twitter, basado en su capacidad de éxito alrededor de la influencia ejercida a otros miembros dentro de las redes sociales, no depende únicamente de la capacidad que tengan en dar comienzo a un debate, alrededor de un determinado tema, sino del marco cultural prevaleciente durante el proceso de interacción entre el líder de opinión y quienes le siguen. Lo antes planteado reviste una crítica de las teorías tradicionales, alrededor de la influencia ejercida desde los medios de comunicación, al no contemplarse en ella un rol desta- 
cado de los usuarios normales (no líderes de opinión). Por ello, que resulta conveniente abordar el tema de la influencia desde una óptica que tenga en cuenta las nuevas capacidades ejercidas por los escenarios digitales actuales, en el que se analicen aspectos como los considerados en este trabajo, en lo que se refiere a identificar los factores que puedan estar incidiendo en la ganancia y pérdida de seguidores, por parte de los considerados líderes de opinión (usuarios más prominentes) en Twitter, desde el impacto que ejerce la publicación de mensajes a los usuarios que les siguen, quienes poseen un rol directo de incidencia en la prominencia o no de los casos aquí analizados, al momento de replicar, comentar, mencionar y compartir lo que se publica en unos usuarios, categorizados, a partir de estudios adelantados por Wu et al. (2011), bajo cuatro subtipos: medios de comunicación, organizaciones, blogueros y celebridades (entre las cuales también están personas vinculadas a la política).

\section{Metodología}

El estudio expuesto en este artículo fue de corte cuantitativo, considerando al usuario de alta "prominencia" (por encima de 50 puntos), según el algoritmo empleado por la herramienta Wefollow (http://wefollow.com/about/score), la unidad de análisis; y tomado como unidades operativas de análisis el perfil del usuario en Twitter y los tweets (mensajes) emitidos por dichos usuarios. La selección en el análisis de los usuarios más prominentes en vez de los usuarios con más seguidores en Twitter se hizo, en vista que el interés en este artículo era establecer un marco de análisis que ayudase a comprender mejor los factores que ayudan a que la población analizada presente en cada uno de los países seleccionados como casos de estudio regional en América Latina y Medio Oriente, respectivamente, niveles significativos de influencia (no necesariamente de seguidores), a partir de estándares establecidos por herramientas de medición online dirigidos a este tipo de usuarios de Twitter.

En cuanto a los usuarios, la muestra se centró en los 20 de usuarios de Twitter de mayor "prominencia" en Colombia, Chile, México, Venezuela, en el caso de los países latinoamericanos; y Egipto, Irán, Libia y Túnez, en el caso del Medio Oriente, cuyos datos fueron tomados durante el primer semestre de 2012. La selección de estos países, para el análisis regional pautado en este trabajo, se hizo con base a los criterios de selección expuestos por Said y Arcila (2013), a saber: la creciente relevancia que han tenido las redes sociales, en especial Twitter, en los diferentes procesos electorales y sociales acaecidos en dichos países desde 2007 hasta la fecha, en la que destacan el posicionamiento de las redes sociales en el ejercicio político en Venezuela, desde 2007 hasta la fecha); el movimiento de la "Ola Verde" en Colombia, durante 2010; así como las diferentes movilizaciones generadas en países como México, Chile y los países del Medio Oriente seleccionados, en torno a lo que se conoce como "Primavera Árabe", reformas educativas y procesos electorales recientes.

En el caso de los mensajes se seleccionaron los 20 últimos Tweets de cada usuario durante el periodo de estudio, obtenido así una muestra final con las dimensiones expuestas en la siguiente gráfica. 


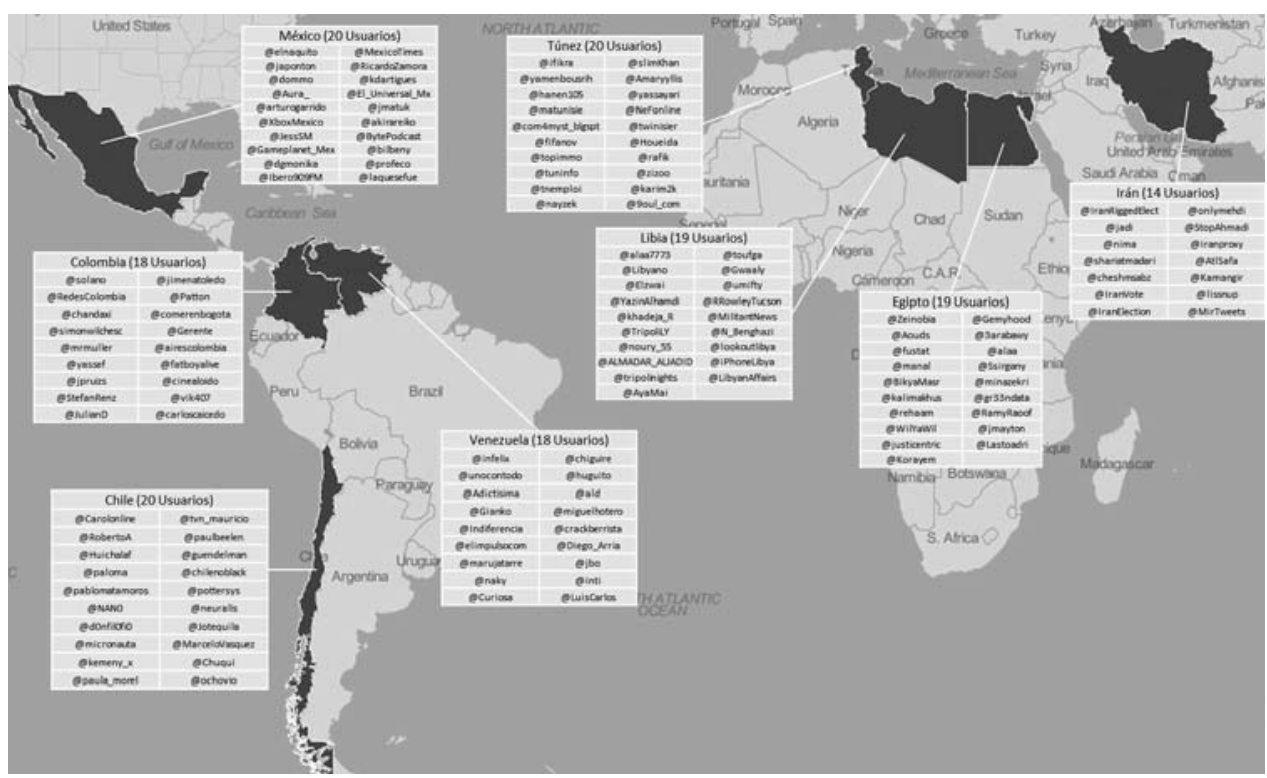

Gráfico 1. Tamaño de la muestra real recopilada. Fuente: elaboración de los autores

Nota: *la diferencia con la muestra ideal corresponde a usuarios que no tuvieron o registraron tweets en el periodo de recopilación de información y fueron retirados del análisis.

Con base a la muestra descrita en el gráfico 1, el estudio orientó su análisis en torno a las siguientes interrogantes: 1) ¿Cuáles son los rasgos que caracterizan los usuarios más prominentes (líderes) de Twitter en las regiones estudiadas?; 2) ¿Cuáles son los rasgos que caracterizan los mensajes más prominentes de Twitter en las regiones analizadas? y 3) ¿Qué factores indicen en la ganancia y pérdida de seguidores, por parte de estos usuarios?

Cada una de estas interrogantes se respondieron a partir de la selección de un conjunto de variables y/o categorías que procuraban dar muestra de las dimensiones generales, de influencia y de participación desde los usuarios seleccionados para el desarrollo de este proyecto, a partir de la identificación de aspectos como: 1) el perfil de uso y social de dichos usuarios; 2) la agenda de contenidos expuesta desde los perfiles de usuarios analizados; 3) el tipo de influencia e impacto ejercido por estos usuarios a otros miembros de Twitter y 4) las redes sociales tejidas alrededor de los mensajes expuestos por este tipo de usuarios.

Cada una de estas dimensiones fueron analizada a partir de la aplicación de la medición de indicadores de tipo cuantitativos ya existentes a partir de las siguientes herramientas de análisis online: buscador avanzado de Twitter, Twerpscan, Tweetstat, Twitalyzer, Tweeteffect, TwitterCounter y RetweetRank.

Los datos e indicadores extraídos, además de valerse de cálculos tomados directamente de Twitter, hacen uso total de los contenidos expuestos en cada uno de los usuarios escogidos para el estudio, o bien, en el caso de RetweetRank y Twerpscan, hace 
un análisis estadísticamente significativo, con un nivel de confianza del 97\% y a $=$ 0,01, de, aproximadamente 12.000 Tweets del total publicado por los usuarios (RetweetRank); y de más del 95\% de los Tweets enviados (Twerpscan).

El análisis de los mensajes extraídos de los usuarios analizados permitió la construcción de una muestra por región analizada con un nivel de confianza del 99\% y a $=0,05$, con una afijación simple en el que se tomaron para análisis los 20 últimos Tweets publicados en cada perfil de los usuarios sujetos de estudio, al momento de levantamiento de la información requerida para el desarrollo de este estudio, durante el período de levantamiento pautado en 2012. Sobre este grupo de mensajes se aplicó la técnica de análisis de contenido para extraer la información necesaria para la consecución de los objetivos de la investigación en términos de identificación del tipo de contendido de los mensajes, su tono y actitud, la interacción en los mensajes con otros usuarios, el abordaje temas o problemáticas sociales y a las agendas particulares de los usuarios, así como la relación de estas características con la ganancia o pérdida de followers.

\section{Resultados}

\subsection{Rasgos generales de los usuarios más prominentes en Twitter de América La- tina y Medio Oriente}

Uno de los primeros rasgos que deseamos remarcar en torno a los usuarios más prominentes de los países analizados en América Latina y el Medio Oriente, es el puntaje medio de influencia que se observaron a partir del análisis regional realizado para cada caso. En general, se aprecia como ambos grupos de usuarios poseen niveles que pudiesen ser considerados medios, entre el grupo de usuarios ubicados en el cuartil con puntaje más elevado considerado por WeFollow, 76 puntos en el caso de los usuarios analizados de América Latina y 72 puntos en los usuarios estudiados del Medio Oriente. Por tanto, en términos generales podemos decir que, a nivel de los países analizados en América Latina y Medio Orienta, los niveles de influencia ejercido por los usuarios estudiados se ubican en una franja media de influencia en Twitter.

En cuanto al género los datos obtenidos en torno a las regiones analizadas nos permiten establecer dos perfiles con claras diferencias a nivel de los usuarios más prominentes analizados en los países tomados como casos de estudio propuesto aquí. En lo que se refiere a América Latina, podemos indicar que se aprecia una amplia presencia de usuarios prominentes masculinos ( $72 \%$ del total de usuarios analizados); mientras que en el caso de los países del Medio Orienta analizados, se observa como los usuarios más influyentes se distribuyen en torno a dos tipos: hombres (49\%) e instituciones $(43 \%)$.

Al momento de identificar a los usuarios más prominentes de los países, procedentes de las regiones consideradas en este trabajo, apreciamos diferencias claras. Mientras que en América Latina, las nacionalidades de estos usuarios se centran en su totalidad a los mismos países analizados (Chile, Colombia, México y Venezuela), en porcentajes similares que van del 23\% al 26\%; mientras que en el Medio Oriente, se aprecia una mayor fragmentación de los usuarios estudiados, según su nacionalidad, a pesar de observarse también que, en su mayoría, se concentran en torno a los países 
de estudio (Egipto, Irán, Libia y Túnez), con el 91,6\% de los usuarios más prominentes analizados, quedando el restante $8,4 \%$ distribuidos en países como Bélgica, Estados Unidos, Francia y Marruecos, por ejemplo.

Sobre el rol que tienen los usuarios más prominentes de las regiones analizadas dentro de Twitter, los datos nos muestran como en su mayoría, tanto en los países estudiados en América Latina (95\% de los usuarios analizados), como en el Medio Oriente ( $100 \%$ de los usuarios analizados), se pueden tipificar como Everyday Users, es decir, usuarios con pequeños círculos de influencia a nivel de otros usuarios en Twitter pero un gran potencial de contacto desde esta red social.

En cuanto a los horarios y días de la semana en los que suelen publicar los casos analizados, tanto en los países de América Latina como en los del Medio Oriente tomados a consideración, se observa un uso mayoritario de toda la franja horaria, al momento de publicar y responder Tweets, por parte de los usuarios más prominentes estudiados ( $82 \%$ y $68 \%$, respectivamente); además de apreciarse un uso más intensivo de Twitter durante los días laborales (lunes a viernes) que durante los fines de semana, en vista que el $76 \%$ de los Tweets publicados por estos usuarios, sin importar la región estudiada se hicieron en estos días.

Al momento de intentar comprender los rasgos generales de los usuarios más prominentes de los países seleccionados de las regiones de estudio (América Latina y Medio Oriente), resulta importante establecer el tipo o perfil que, a nivel general, poseen este grupo de usuarios analizados. Según los datos obtenidos, se aprecia una variación en torno al tipo de usuario predominante dentro de los países analizados dentro de las regiones consideradas aquí. Mientras que en América Latina el 57\% de los usuarios prominentes son de tipo profesional o institucional, es decir, vinculados a empresas, áreas de actuación u ocupaciones socialmente relevantes (artistas, políticos, empresarios y periodistas, por ejemplo); en el caso de los países del Medio Oriente, el peso ( $57 \%$ de los casos analizados) dentro de los usuarios más promitentes lo tienen aquellos con un perfil personal, es decir, cuentas que no poseen relación alguna dentro de su zona biográfica con empresas o labores profesionales ejecutadas por quienes los dueños de estos perfiles. Estos datos nos permite ver uno de los rasgos de mayor diferencia entre países y regiones de estudio: el uso y oportunidades que brinda este microblogs para el reforzamiento de los líderes de opinión tradicionales (caso América Latina) y de un nuevo tipo de líderes de opinión online (invisibles desde los medios de comunicación tradicionales) como se observa en el caso de los países del Medio Oriente, en general.

Otro aspecto a ser tenido en cuenta es el idioma empleado por los usuarios más prominentes de Twitter en los países y regiones tomadas como casos de estudio de este trabajo, lo cual nos ayudará a ver el tipo de lenguaje empleado por estos para el contacto con sus seguidores (followers) y seguidos (followings) dentro de este Micloblogs, así como el carácter global de éstos, no solo determinado por la nacionalidad que poseen, sino también por la lengua empleada para hacer trascender sus mensajes o publicaciones a usuarios procedentes de otros países y regiones, con idiomas ajenos a los casos estudiados, por ejemplo. En torno a lo aquí expuesto, vemos cómo, en el caso de los países analizados de América Latina, los usuarios más prominentes ter- 
minan centrando su presencia en Twitter, a través de mensajes emitidos en el idioma oficial (castellano), lo que les da una relevancia local, nacional o regional, en el mejor de los casos; mientras que en los casos estudiados en el Medio Oriente, estos usuarios hacen uso mayoritario del inglés ( $85 \%$ de los mensajes analizados en este trabajo) como lengua empleada para establecer su comunicación desde este Microblog; quedando el árabe o persa (lengua oficial en estos países) y el francés (lengua secundaria en países como Túnez, en vista de su pasado colonial con Francia) con un bajo nivel de uso ( $8 \%$ y $7 \%$ respectivamente).

Por último, a partir de las medias $(\mu)$ observadas en la tabla 1 , vemos cómo los usuarios más prominentes analizados en los países de América Latina, se caracterizan por: estar mejor ubicados dentro del ranking en Twitter a nivel mundial; un mayor tiempo en Twitter (poco más de 3 años en promedio, aproximadamente); un mayor número de followers (seguidores) y de followings (usuarios que siguen); una menor tendencia de ganancia de seguidores al mes, aunque presentan un promedio de Tweets publicados similar a lo observado en el caso de los usuarios más prominentes de los países tomado para estudio en el Medio Oriente, caracterizados a su vez por: una peor ubicación dentro del ranking de usuarios en Twitter; una presencia relativamente nueva dentro de este Microblog (poco más de 2 años en promedio, aproximadamente); un menor número de seguidores y de usuarios que siguen; aunque con un mayor impacto en lo que se refiere a la tendencia mensual observada en lo que se refiere al número de seguidores ganados dentro de este escenario digital.

Tabla 1: Datos generales de perfil de usuarios más prominentes analizados en América Latina y el Medio Oriente. Fuente: elaboración de los autores. N=148 usuarios analizados

\begin{tabular}{|l|l|l|l|l|l|l|l|l|}
\hline \multirow{2}{*}{\multicolumn{1}{|c|}{ Indicador }} & \multicolumn{5}{|c|}{ América Latina } & \multicolumn{4}{c|}{ Medio Oriente } \\
\cline { 2 - 9 } & Media $(\boldsymbol{\mu})$ & Desv. típ. & $\begin{array}{c}\text { Mínim } \\
\mathbf{0}\end{array}$ & Máximo & Media $(\boldsymbol{\mu})$ & Desv. típ. & Mínimo & Máximo \\
\hline $\begin{array}{l}\text { Ranking que posee } \\
\text { el usuario en Twitter } \\
\text { a nivel mundial }\end{array}$ & $68.176,32$ & $76.275,98$ & 541,00 & $486.798,00$ & $135.242,54$ & $114.244,17$ & $14.743,00$ & $481.267,00$ \\
\hline $\begin{array}{l}\text { Tiempo del usuario } \\
\text { en Twitter } \text { en meses }\end{array}$ & 37,89 & 10,38 & 24,00 & 48,00 & 26,54 & 12,40 & 4,00 & 48,00 \\
\hline Número de followers & $38.510,91$ & $109.196,54$ & 547,00 & $790.484,00$ & $5.635,94$ & $8.697,75$ & 3,00 & $39.619,00$ \\
\hline $\begin{array}{l}\text { Número de } \\
\text { followings }\end{array}$ & $8.901,13$ & $21.994,48$ & 102,00 & $113.018,00$ & $1.174,72$ & $2.670,42$ & 2,00 & $20.087,00$ \\
\hline $\begin{array}{l}\text { Número de Tweets } \\
\text { generado por usuario }\end{array}$ & $28.820,26$ & $38.764,93$ & 52,00 & $250.205,00$ & $16.738,71$ & $26.276,02$ & 1,00 & $145.168,00$ \\
\hline $\begin{array}{l}\text { Tendencia a corto } \\
\text { plazo de followers } \\
\text { en usuario Twitter } \\
\text { (mensual) }\end{array}$ & 1377,513 & 2230,07241 &, 00 & 14057,00 & 1903,5417 & 3671,94726 &, 00 & 22256,00 \\
\hline
\end{tabular}

Lo expuesto hasta ahora nos permite establecer un marco general alrededor de los usuarios prominentes de ambas regiones estudiadas, al menos en los países tomados para este análisis, en el que, pese a observarse semejanzas entre sí, también nos permite establecer diferencias que resultan claves al momento de entender el comportamiento de este tipo de usuarios en países y regiones con claras diferencias socio-culturales y política, en especial durante el tiempo de levantamiento de la información requerida para el desarrollo de este estudio, marcada por la creciente mo- 
vilización social realizada bajo el amparo de las redes sociales (ejemplo: la Primavera Árabe y Movimiento estudiantil a favor de la Calidad Educativa en Chile, por ejemplo); los elevados controles estatales ejercidos a los medios de comunicación tradicionales, bajo la puesta en marcha de leyes (ejemplo de ello es la Ley de Responsabilidad Social en Radio y Televisión puesta en marcha en 2005 y reformada en 2010 en Venezuela); el impacto que ha traído consigo la creciente presencia de los escenarios digitales como Twitter en el ejercicio de campañas políticas y campañas electorales en los diferentes países analizados (tal es el caso del Movimiento YoSoy132 en México, el creciente uso de Twitter para el seguimiento electoral en Venezuela desde 2009, la creciente denuncia por irregularidades durante la elección de Mahmud Ahmadineyad en Irán en 2009, o el impacto que ejerció Facebook y Twitter en el desarrollo de la campaña electoral para la presidencia de Colombia en 2010); o bien como resultado de las diferentes formas de gobierno existentes en los diferentes países analizados, en especial en los países tomados de referencia en el Medio Oriente, caracterizados por una alta presencia de dictaduras (caso Egipto); con un alto nivel de conflictividad civil y social, traducida en lucha armada entre quienes ejercen el poder y ciudadanos rebeldes a éste (caso de Libia); o con un alto componente religioso en él (caso Irán, al ser una República Islámica), que restringen el acceso de los ciudadanos a una mayor diversidad de canales tradicionales de debate a nivel social. Lo aquí señalado trae consigo, no solo, cambios destacados en cómo pueden estar siendo usado socialmente escenarios digitales como Twitter, sino también la variabilidad que puede existir en torno a los factores o rasgos que inciden para la prominencia o no de usuarios desde este Microblog y en las ganancia o pérdida de los usuarios analizados, de acuerdo con la forma como participan activamente desde este escenario.

Si cruzamos los datos obtenidos de los usuarios más prominentes de los países de América Latina y el Medio Oriente considerados para el desarrollo de este trabajo, según la ocupación, el tipo de relevancia social, y el área de actuación de estos, en general (Gráfico 2), observamos cómo el perfil de este tipo de usuarios varía significativamente. Es así como, en países como Egipto, Irán y Venezuela, los usuarios más prominentes se enmarcan en áreas de actuación vinculadas con la política y las finanzas o empresas, con una relevancia social de tipo internacional; mientras que en el caso de Libia y México, estos suelen estar más afines a los medios de comunicación, periodismo y el mundo académico internacional; en lo que se refiere a Chile y Túnez, este tipo de usuarios suelen poseer una relevancia más nacional, enmarcados más en ocupaciones afines al ámbito empresarial; mientras que Colombia, último país analizado en este trabajo, el perfil de los usuarios más prominentes se ubican en áreas de actuación y ocupaciones vinculadas con las artes, la moda y el espectáculo. 


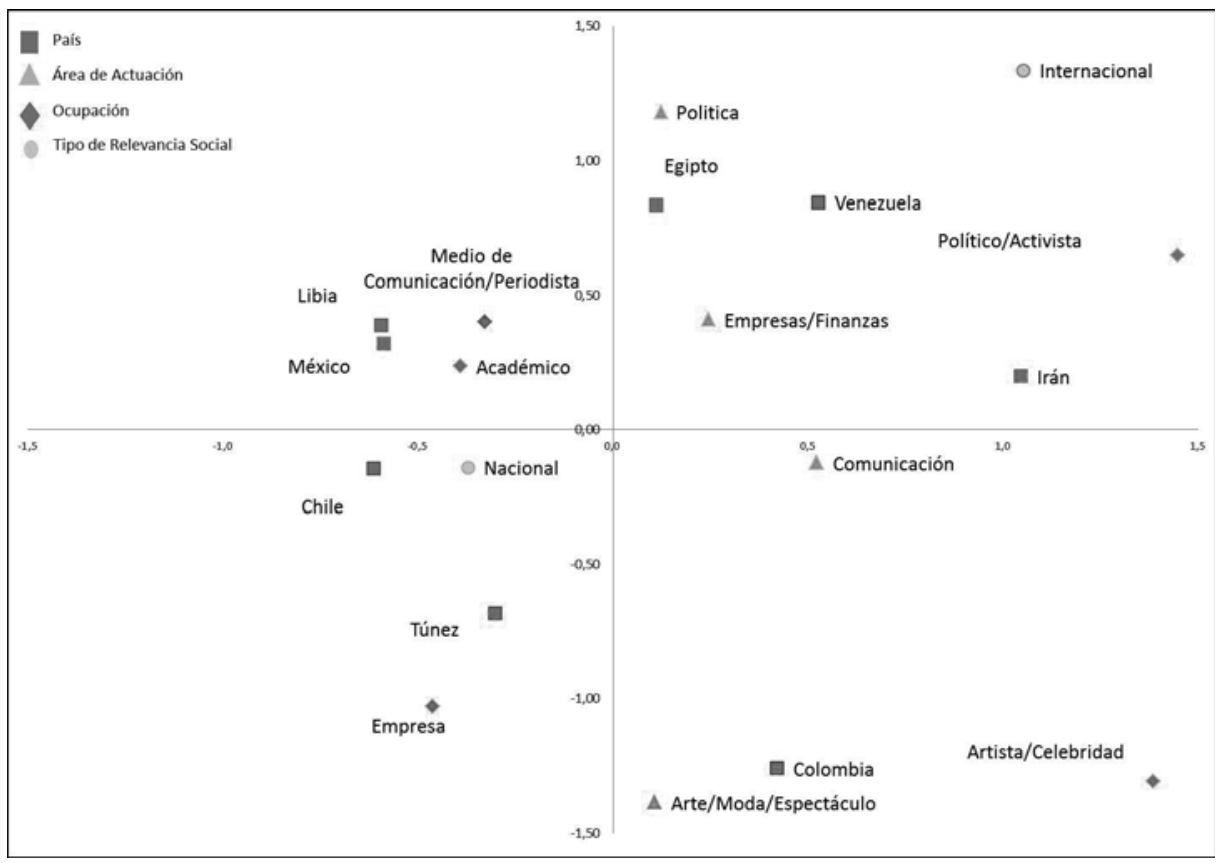

Gráfico 2: Perfil de los usuarios según País, Ocupación, Tipo de Relevancia Social y Área de Actuación. Fuente: Elaboración de los autores

\subsection{Características de los mensajes de los usuarios más prominentes de Twitter}

En cuanto a las características de los mensajes publicados por los usuarios analizados en este artículo ( $\mathrm{N}=1.223$ mensajes o Tweets analizados en total), tanto en el caso de América Latina como en el Medio Oriente, apreciamos cómo, a pesar de haber una mayor presencia de mensajes de tipo personal (saludos, respuestas a otros usuarios, ideas sin carga de crítica u opinión de índole político o social), con el $68,4 \%$ y $56 \%$ de la totalidad de los mensajes analizado; también se observa una clara diferencia entre el tipo de contenido expuesto en los mensajes de estos usuarios de ambas regiones, al ver cómo en el caso de los casos analizados, provenientes de los países del Medio Oriente, tienen un especial peso también los mensajes de tipo noticioso y de información de acontecimientos sociales, económicos o políticos $(30,2 \%$ entre la totalidad de mensajes analizados), en comparación con otros tipos de contenidos tipificados en este trabajo: mensajes de tipo promocional $(7,9 \%)$, mensajes de tipo crítica u opinión $(3,9 \%)$ e institucionales $(2.3 \%)$. En el caso de los usuarios más prominentes en los países de América Latina seleccionados se aprecia niveles similares entre los mensajes de tipo institucional $(10,9 \%)$, mensajes de tipo crítica u opinión $(9,6 \%)$, mensajes noticiosos y de información $(8,2 \%)$ y los mensajes de tipo promocional, cuyo peso es menor en el conjunto de Tweets analizados en dichos usuarios (1,9\%).

En lo que se refiere a la mención a otros usuarios que hacen los casos analizados en América Latina y el Medio Oriente, se aprecia cómo los usuarios más prominentes de la primera región son más proclives $(66,9 \%$ de los mensajes analizados men- 
cionaban a otro usuario) a vincular dentro de sus Tweets a otros miembros de este $\mathrm{Mi}$ croblog en sus mensajes que en el caso del Medio Oriente (51,5\% de los mensajes analizados mencionaban a otro usuario).

Al momento de medir los niveles de alusión a temas o problemáticas sociales, los usuarios más prominentes analizados de los países del Medio Oriente, mostraron un mayor nivel de sensibilidad o interés en la publicación de mensajes con un alto componente social (33\% de los mensajes analizados), en comparación el caso de los usuarios estudiados en América Latina, cuyo Tweets analizados resultaron ser, en su mayoría, más alejados a este tipo de temas (solo el 19,7\% de los mensajes analizados eran alusivos a temas o problemáticas sociales). Los datos mostrados nos muestra un marco expositivo de mensajes, desde los usuarios más prominentes, posiblemente impactados por los escenarios políticos y sociales vinculados a cada uno de los países y regiones analizados, lo cual haría que, por ejemplo, Twitter se pudiese estar convirtiendo en una válvula de escape social, con una doble función: en el caso de los países del Medio Oriente, para hacer ver o abordar temas sociales que puedan resultar opacados bajo el contexto social, político y económico existente en ellos y como recurso de evasión de los diferentes problemas sociales, políticos y económicos existentes a nivel diario entre quienes siguen y hacen prominentes a los usuarios analizados en este trabajo. Lo antes expuesto, se aprecia mejor cuando observamos la cercanía que presentan los usuarios más prominentes de los países analizados por regiones tratadas, con base a las expresiones o términos de mayor empleo en ellos: mientras que en el Medio Oriente se observa una mayor cercanía en el uso de terminología que alude a temas políticos de actualidad de carácter nacional (propios países) o regional (otros países vecinos) e internacionales (caso Estados Unidos); en América Latina, los conceptos y términos con mayores niveles de uso son aquellos que versan más próximos a temas deportivos (mundial), tecnológicos, personales (saludos, comentarios), en los casos de Colombia, México y Venezuela, siendo en el caso de los usuarios más prominentes de Chile quienes abordan más temas de actualidad nacional (como es el caso de las manifestaciones y movilizaciones ocasionadas por la huelga educativa que tuvo lugar en dicho país, durante el período de medición hecho para el desarrollo de este trabajo).

En cuanto a la profundidad del mensaje o Tweet publicado por los usuarios más prominentes, los datos obtenidos nos permiten ver cómo, en términos generales, en el caso de América Latina, los mensajes publicados por los usuarios analizados no poseen links en ellos $(27,6 \%)$, o bien se hace mención a otros usuarios o temas, a través de los hashtags (54,6\%); mientras que los mensajes con links apenas representa el 17,2\% y los mensajes replicados, provenientes de otros usuarios (Retweets) apenas el 0,5\%, de la totalidad de mensajes analizados en este caso. En lo que se refiere a los usuarios analizados en los países del Medio Oriente, se observa un mayor nivel de uso de los mensajes con links $(32,4 \%)$, en comparación con los mensajes en los que se hace mención a otros usuarios o temas $(42,9 \%)$, los mensajes sin links $(22,1 \%)$ y mensajes replicados $(2,6 \%)$. Los datos aquí mostrados nos marcan un contexto de mayor presencia de niveles de profundidad de los mensajes publicados por los usuarios más prominentes de los países del Medio Oriente seleccionados, en comparación con los estudiados en 
América Latina; mientras que, en el caso de estos últimos, se observa un mayor nivel de generación de mensajes propios y aquellos orientados a generar un mayor nivel de interacción con otros usuarios, aludiendo a estos directamente o bien.

Un último aspecto resta por describir para caracterizar los mensajes analizados por parte de los usuarios analizados en este artículo, según las regiones abordadas: la actividad del mensaje. Al respecto, los datos nos permiten apreciar una tendencia más proclive en el caso de los usuarios más prominentes de los países de América Latina a la generación de mensajes con actitud positiva $(81,7 \%)$ y negativa $(7,1 \%)$, que en el caso de lo observado en los mensajes de los usuarios estudiados de los países del Medio Oriente, en el que los mensajes con actitud positivo llegan al $69,2 \%$ de los mensajes analizados en este trabajo, mientras que los mensajes con actitud negativa apenas llega a $0,6 \%$ de la totalidad de mensajes analizados. En lo que se refiere a los mensajes con actitud neutra (mensajes sin carga valorativa), los mensajes analizados, relacionados con los usuarios del Medio Oriente, tienen un mayor peso $(30,2 \%$ del total analizado) en comparación con América Latina, en el que apenas llegaron a identificarse dentro de la muestra el $11,2 \%$ de mensajes con este tipo de actitud.

\subsection{Factores que inciden en la ganancia y/o pérdida de followers de los usuarios más prominentes de Twitter}

Tal como se mencionó en el apartado metodológico, se pretende establecer factores de mayor incidencia sobre la variabilidad de Followers, a partir de las características de usuarios y mensajes; para lo cual se desarrolló de un modelo de regresión logística que permitió determinar la probabilidad de que un usuario gane y mantenga o pierda sus Followers a partir de cada una de las variables recopiladas de las diferentes herramientas de análisis, tomando como variable explicativa el estado, condición, movimiento neto del número Followwers de usuarios y mensajes generada por la herramienta TweetEfect.

Esta variable se operacionalizó como dicotómica en las siguientes unidades: 1) Gana o Mantiene el número de Followers o 2) Pierde Followers con el fin de evitar la aparición de casillas con bajas frecuencias que pudiesen afectar la consistencia de los resultados. Este procedimiento se aplicó igualmente a aquellas variables independientes que requirieron dicho ajuste.

Una ajustadas y transformadas las escalas de las variables se procedió a la definición del modelo de regresión, esto se realizó en dos pasos el primero consistió en evaluar modelos individuales con cada variable predictiva, con el fin de evaluar el aporte individual de cada una de estas. El segundo consistió en evaluar del modelo introduciendo las variables por paso por paso evaluando en para cada paso la distribución casos clasificados de forma correcta determinando su especificidad y sensibilidad dejando de lado aquellas variables con aportes no significativos a la variabilidad de la variable dependiente.

La tabla 2 presenta los resultados del modelo final ${ }^{1}$ para las características de cada uno de los grupos de usuarios analizados, según las regiones estudiadas en este trabajo.

1 La evaluación de la bondad de ajuste del modelo se realizó a través de la deviación (2LL), el R2 de Cox -Snell, el R2 Nagelkerke y la prueba de Hosmer y Lemeshow. 
En estas se incluyen los coeficientes de regresión (B), el error estándar de estimación (E.T), el valor del estadístico W de Wald, con los grados de libertad (g.l.) y su nivel de significancia (Sig.) y el riesgo (Exp (B)) de cada variable con sus respectivos intervalos de confianza.

Tabla 2. Resultados del Modelo de Regresión de usuarios más prominentes analizados, según región. Fuente: elaboración de los autores

\begin{tabular}{|c|c|c|c|c|c|c|c|c|}
\hline \multirow{2}{*}{ Región } & \multirow{2}{*}{ Rasgos } & \multirow{2}{*}{ Indicador } & \multicolumn{6}{|c|}{ Variables en la Ecuación } \\
\hline & & & B & E.T. & Wald & gl & Sig. & $\operatorname{Exp}(\mathbf{B})$ \\
\hline \multirow{8}{*}{$\begin{array}{l}\text { América } \\
\text { Latina }\end{array}$} & \multirow{5}{*}{$\begin{array}{l}\text { Características de } \\
\text { los mensajes** }\end{array}$} & Presencia de Mention (1) & ,630 & ,267 & 5,545 & 1 & ,019 & 1,877 \\
\hline & & Actitud del Mensaje & & & 19,149 & 2 & , 000 & \\
\hline & & Positivo (1) & $-2,050$ & ,469 & 19,142 & 1 &, 000 &, 129 \\
\hline & & Neutro (2) & $-1,827$ &, 565 & 10,442 & 1 &, 001 &, 161 \\
\hline & & Constante & ,632 & ,470 & 1,810 & 1 & , 178 & 1,882 \\
\hline & \multirow{3}{*}{$\begin{array}{l}\text { Características de } \\
\text { los Usuarios* }\end{array}$} & Ranking en el País &,- 196 & ,066 & 8,686 & 1 & ,003 &, 822 \\
\hline & & Tiempo en Twitter &, 058 & ,030 & 3,831 & 1 &, 050 & 1,060 \\
\hline & & Constante & 2,288 & 1,066 & 4,609 & 1 & ,032 & 9,857 \\
\hline \multirow{9}{*}{$\begin{array}{l}\text { Medio } \\
\text { Oriente }\end{array}$} & \multirow{7}{*}{$\begin{array}{l}\text { Características de } \\
\text { los mensajes** }\end{array}$} & $\begin{array}{l}\text { Tipo de Contenido del } \\
\text { Mensaje }\end{array}$ & & & 15,371 & 2 &, 000 & \\
\hline & & Mensaje Personal (1) &,- 524 & , 198 & 7,003 & 1 & ,008 &, 592 \\
\hline & & Mensaje Institucional (2) & ,410 & ,256 & 2,562 & 1 & 109 & 1,507 \\
\hline & & Actitud del Mensaje & & & 6,476 & 2 & ,039 & \\
\hline & & Positivo (1) &,- 537 & 1,024 & ,275 & 1 & ,006 &, 584 \\
\hline & & Neutro (2) & $-1,027$ & 1,037 & ,981 & 1 & ,002 & ,358 \\
\hline & & Constante & 297 & 1,036 & 082 & 1 & ,775 & 1,346 \\
\hline & \multirow{2}{*}{$\begin{array}{l}\text { Características de } \\
\text { los Usuarios* }\end{array}$} & Tiempo en Twitter & , 145 & ,038 & 14,872 & 1 & ,000 & 1,156 \\
\hline & & Constante & $-2,447$ & 819 & 8,932 & 1 & ,003 &, 087 \\
\hline
\end{tabular}

$*$-2 log de la verosimilitud $=148,822 ; \mathrm{R}$ cuadrado de Cox y Snell= 0,094; R cuadrado de Nagelkerke $=0,125$

** $-2 \log$ de la verosimilitud $=1960,835 ; \mathrm{R}$ cuadrado de Cox y Snell=0,008; $\mathrm{R}$ cuadrado de Nagelkerke $=0,011$

Tipo de Contenido: Mensaje Personal =1; Otro = 0; la categoría Otro corresponde a Mensaje Noticioso/Informativo o Mensaje Institucional/Profesional. Actitud: $1=$ Positivo, $0=$ Negativo-Neutro. $\mathrm{N}=1.223$ Tweets

Lo observado en la tabla 2, nos permite ver como la ganancia y/o pérdida de seguidores (followers) en Twitter, en el caso de América Latina, tendrá mayor probabilidad los casos estudiados cuando hacen mención expresa de otros usuarios de esta red social, exponen o publican mensajes polémicos, en los que se plasmen actitudes negativas o críticas, se ubican en niveles superiores dentro del ranking de esta red social y tienen más tiempo en ella. En el caso del Medio Oriente, las probabilidades de ganancia y/o pérdida de seguidores (followers) en Twitter por los usuarios más prominente analizados variará en comparación a lo antes expuesto, ya que será mayor cuando se aluden o publican mensajes de corte más institucionales y/o profesionales, se exponen o publican mensajes polémicos, en los que se plasmen actitudes negativas o críticas, y estos tienen mayor tiempo de permanencia en esta red social. 


\section{Discusión y Conclusiones}

Los datos expuestos en este trabajo, no solo, ayudan a reafirmar el impacto que están trayendo consigo el auge de las redes sociales, en torno a la relación que entre los líderes de opinión y la audiencia, así como el conjunto de transformaciones que han sido consideradas por autores referidos en este trabajo, como por ejemplo Jensen (2009) y Marwick y Boyd (2011), Said y Arcila (2011), Claes, Osteso y Deltell (2013); sino también nos permite establecer un marco referencial alrededor de los rasgos que guardan común o no los usuarios más prominentes de Twitter en América Latina y el Medio Oriente, al menos en el caso de los países tomados como casos de estudio para el estudio.

Los planteamientos hechos por Lazarsfeld, Berelson y Gaudet (1979), más que perder vigencia, se complejizan; dando paso a nuevos referentes conceptuales, como la propuesta por Jensen (2009), las cuales ayudan a la actualización del debate alrededor de la influencia y contactos que ejercen los líderes de opinión con el resto de miembros de nuestras sociedades, ante el auge de las redes sociales. Bajo este contexto actual, los nuevos escenarios digitales están favoreciendo la reafirmación de los espacios de influencia de los líderes de opinión tradicionales, en los diferentes países; sino que se convierten, dependiendo de la región y país (en nuestro caso ello es más palpable en el caso de los países del Medio Oriente), en ventana para la aparición de nuevos líderes de opiniones de escala mundial, enmarcados bajo las tipologías de usuarios destacadas por Wu et al. (2011), las cuales variarán de acuerdo con los diferentes rasgos que dimos cuenta, al momento de exponer los principales rasgos que caracteriza a los usuarios más prominentes estudiados.

Los datos además nos reafirman lo indicado por Túñez y Sixto (2012), al momento de remarcar los diferentes rasgos que les caracterizan como poseedores de altos niveles de participación en Twitter, por parte de los usuarios más prominentes analizados en América Latina y el Medio Oriente, como resultado del perfil y frecuencia (día y hora de publicación en esta red social), que se pudieron apreciar durante el análisis efectuado, en cada uno de los casos aquí considerados.

En cuanto a la capacidad de generación ciberactivictas, con que los usuarios más prominentes analizados en América Latina y Medio Oriente, desde los usuarios que les siguen (Olorunnisola y Martin, 2013), los datos hacen que lo destacado por estos autores, no se ejerza de forma homogénea dentro de las redes sociales; siendo los países del Medio Oriente, donde se perciben mayores niveles de promoción de ello, a través de las áreas de actuación y la alusión de temas o problemáticas sociales promovidos desde los usuarios analizados. Por tanto, reafirmaríamos lo antes expuesto, en cuanto al empleo que está teniendo Twitter, al menos en el caso del Medio Oriente, como potencial válvula de escape social para hacer ver o abordar temas sociales que puedan resultar opacados bajo el contexto social, político y económico existente en ellos; mientras que en el caso de América Latina, su función pareciera estar más avocada a la evasión de los diferentes problemas sociales, políticos y económicos existentes a nivel diario entre quienes siguen y hacen prominentes a los usuarios analizados en este trabajo.

Lo expuesto en este artículo nos permite reafirmar la capacidad que tienen las redes sociales, en nuestro caso Twitter, en torno a lo expuesto antes por Golder y Macy 
(2011), ya que tanto en América Latina como en el Medio Oriente se apreciaron un conjunto de rasgos en los mensajes publicados por los usuarios analizados, en los que queda palpable la conformación de nuevos mecanismos de participación e interacción entre el líder online y quienes les siguen; en el que las relaciones emocionales (a través de la publicación de mensajes personales) y la alusión directa de usuarios, ejercen un rol muy importante. Ello, sin dejar de lado lo indicado, en torno a la mayor presencia de niveles de profundidad de los mensajes publicados en los casos del Medio Oriente que en América Latina.

Finalmente, los datos también nos ayudan a reafirmar lo expuesto por Bakshy et al. (2011), Cha et al. (2010) y Watts (2007), en cuanto a los factores que pueden estar incidiendo en la capacidad de influencia entre los usuarios más prominentes en los países y regiones analizadas, al apreciarse en ellos, no solo, aspectos que juegan en la ganancia y pérdida de seguidores, relacionados con indicadores directamente vinculados con la ubicación o presencia de estos líderes en Twitter; sino también, el rol destacado que tienen elementos como la capacidad de interacción con otros usuarios, el tipo de contenido publicado, y la actitud plasmada en los mensajes, al momento de ejercer una mayor o menor influencia dentro de esta red social.

\section{Referencias bibliográficas}

BAKSHY, Eytan; HOFMAN, Jake; MASON, Winter; \& WATTS, Duncan (2011): "Everyone's an influencer: quantifying influence on twitter". Proceedings of the fourth ACM international conference on Web search and data mining: http://misc.si.umich.edu/media/papers/wsdm333w-bakshy.pdf [Consulta: 12 de enero de 2014].

CASTELLS, Manuel (2009): Comunicación y poder. Madrid, Alianza Editorial.

CLAES, Florencia; OSTESO, José y DELTELL, Luis (2013): "Audiencias televisivas y líderes de opinión en Twitter. Caso de estudio: El Barco". Estudios sobre el mensaje periodístico. Vol. 19, núm. 1, pp. 347-364. DOI: 10.5209/rev_ESMP.2013.v19.n1.42526. Madrid, Servicio de Publicaciones de la Universidad Complutense.

DELTELL, Luis; CONGOSTO, Mariluz; CLAES, Florencia; y OSTESO, José (2013): "Identificación y análisis de los líderes de opinión en Twitter en torno a Hugo Chávez". Revista Latina de Comunicación Social: http://www.revistalatinacs.org /068/paper/997_UCM/31_Deltell.html [Consulta: 20 de enero de 2014].

CHA, Meeyoung; HADDADI, Hamed; BENEVENUTO, Fabrício; \& GUMMADI, Krishna (2010): "Measuring user influence in Twitter: The million follower fallacy". Proceedings of the ICWSM: http://snap.stanford.edu/class/cs224w-readings/cha10influence.pdf [Consulta: 15 de diciembre de 2013].

ESCOBAR, Arturo (2007): “Actors, Networks, and New Knowledge Producers: Social Movements and the Paradigmatic", en SOUSA SANTOS, Boaventura: Cognitive Justice in a Global World. Lanham, Rowman and Littlefield, pp. 273-294. 
GARCÍA-DE-TORRES, Elvira; YEZERS'KA, Lyudmila; ROST, Alejandro; CALDERÍN, Mabel; EDO, Concha; ROJANO, Miladys; SAID-HUNG, Elias; JERÓNIMO, Pedro; ARCILA, Carlos; SERRANO-TELLERÍA, Ana; SÁNCHEZBADILLO, Jorge y CORREDOIRA, Loreto (2011): "Uso de Twitter y Facebook por los medios iberoamericanos". El profesional de la información, vol. 20, núm. 6, pp. 47-53. Barcelona, Universidad de Barcelona. DOI: http://dx.doi.org /10.3145/epi.2011.nov.02

JENSEN, Klaus (2009): “Three-step flow”. Journalism, num. 10, pp. 335-337. Estados Unidos, Universidad de Pensilvania. DOI: http://dx.doi.org/10.1177 /1464884909102594

JURIS, Jeffrey (2005): “The New Digital Media and Activist Networking within AntiCorporate Globalization Movements". The Annals of the American Academy of Political and Social Science, vol. 597, pp. 189-208. USA, SAGE. DOI: http://dx.doi.org/10.1177/0002716204270338.

KATZ, Elihu y LAZARSFELD, Paul (1979): La influencia personal. El individuo en el proceso de comunicación de masas. Barcelona, Hispano Europea.

PARK, Chang (2013): "Does twitter motivate involvement in politics? tweeting, opinion leadership, and political engagement". Computers in Human Behavior, vol. 29, num. 4, pp. 1641-1648. Amsterdam, Elsevier Science Publisher. DOI: http://dx.doi.org/10.1016/j.chb.2013.01.044

MARWICK, Alice y BOYD, Danah (2011): “To See and to Be Seen: Celebrity practice on Twitter". Convergence: The International Journal of Research into New Media Technologies, num. 17, pp. 139-157. UK, University of Suderland. DOI: http://dx.doi.org/10.1177/1354856510394539

MILLER, Daniel \& SLATER, Don (2000): The Internet: An ethnographic approach. UK, Berg.

MORAES, Natalia (2005): "Internet y ciberespacio en el estudio de comunidades diaspóricas: análisis de una experiencia". Comunicación presentada en el Simposio Antropología de los Media del X Congreso de Antropología organizado por la Federación de Asociaciones de Antropología del Estado Español (Sevilla, Septiembre de 2005). Disponible en el archivo del Observatorio para la CiberSociedad en: http://www.cibersociedad.net/archivo/articulo.php?art=208 [Consulta: 12 de octubre de 2013].

OLORUNNISOLA, Anthony \& MARTIN, Brandie (2013): "Influences of media on social movements: problematizing hyperbolic inferences about impacts". Telematics and Informatics, num. 30, pp. 275-288. Amsterdam, Elsevier Science Publisher: http://www.sciencedirect.com/science/article/pii/S0736585312000226

TOFFLER, Alvin (1980): The Third Wave. USA, Morrow.

ROGERS, Everett (1995): Diffusion of innovations. New York, Free Press.

TÚÑEZ, Miguel y SIXTO, José (2011): "Redes sociales, política y Compromiso 2.0: la comunicación de los diputados españoles en Facebook". Revista Latina de Co- 
municación Social, $\mathrm{n}^{\mathrm{o}}$ 66. http://www.revistalatinacs.org/11/art/930_Santiago/09_Tunez.html [Consulta: 14 de febrero de 2013].

UGARTE, David de (2011): El poder de las redes. Bilbao, Biblioteca de las Indias Electrónicas: http://lasindias.com/el-poder-de-las-redes [Consulta: 20 de marzo de 2012].

WATTS, Duncan y DODDS, Peter (2007): “Influentials, networks, and public opinion formation", en Journal of Consumer Research, vol. 34, pp. 441-458. USA, University of Chicago Press. http://www.jstor.org/stable/10.1086/518527

WELLMAN, Barry (2001): "Physical place and cyberplace". International Journal of Urban and Regional Research, vol. 25, num. 2, pp. 227-52. USA, Wiley

WU, Shaomei; HOFMAN, Jake; MASON, Winter \& WATTS, Duncan (2011): "Who says what to whom on twitter", en Proceedings of the 20th international conference on world wide web: https://iriss.stanford.edu/sites/all/files/dsi/Duncan\%20Study \%201.pdf [Consulta: 1 de diciembre de 2013]. 